\title{
Öl vom arktischen Ozean
}

Erst die sogenannte Ölkrise im Winter 1973/74 gab grünes Licht zur Verwirklichung eines der umstrittensten und ehrgeizigsten Vorhaben neuerer Zeit, dem Bau einer Rohölpipeline quer durch die Halbinsel Alaska von den Ölfeldern an der Prudhoe Bay zum $1200 \mathrm{~km}$ südlich liegenden Hafen Valdez.

Im Widerstreit der Meinungen um die Notwendigkeit setzten sich schließlich diejenigen politischen Kräfte durch, die trotz ökologischer Bedenken das Projekt als wichtigen Beitrag im Rahmen der Autarkiebestrebungen der USA werteten.

Die 1967 entdeckten Vorkommen belaufen sich nach bisherigen Schätzungen auf 15,3 Milliarden hl und könnten damit etwa $12 \%$ des amerikanischen Bedarfs decken, wobei mit einer Förderdauer von 30 Jahren gerechnet wird. Bereits 1970 formten die Mineralölkonzerne Exxon und BP ein Konsortium zum Bau der Rohrleitung. Andere Gesellschaften schlossen sich an und bildeten die "Alyeska Pipeline Service Company» als Dachorganisation für Planung, Bau und Betrieb der Anlagen. Die Auflagen des National Environmental Protection Act von 1970 verzögerten jedoch den Baubeginn bis 1974, so daß mit der eigentlichen Rohrverlegung erst am 27. März 1975 begonnen werden konnte. Die Gesamtkosten sind mit 6,375 Milliarden Dollar veranschlagt, das erste Öl soll im Herbst 1977 fließen.

Zwar zählt die Alaska-Rohrleitung weder hinsichtlich ihrer Länge noch ihrer Transportkapazität von 319 Millionen Liter täglich zu den größten Projekten dieser Art, Bodenbeschaffenheit, Tektonik und klimatische Besonderheiten werfen jedoch Probleme auf, die es beim Rohrleitungsbau bisher nicht zu überwinden galt. Einmal liegt die Pipeline im Bereich der zirkumpazifischen Bruchzone, einem Gebiet starker Erdbebentätigkeit, wo tektonische Verschiebungen während der Lebensdauer der Anlagen möglich sind. Zum andern erschwert der nördlich der Brooks-Range vorherrschende Permafrostboden durch Solifluktion, Frostschub und Frosthub die Verlegung.

Aus den Forderungen nach einer möglichst kurzen Leitung zu einem ganzjährig eisfreien Hafen unter Umgehung erdbebengefährdeter Verwerfungszonen und instabiler Frostböden kristallisierte sich schließlich folgende Linienführung heraus: Von den Ölfeldern an der Prudhoe-Bay folgt die Rohrleitung dem Sagavanirkok Fluß und den Atigun Tälern durch baumlose Tundra, ehe sie am 1400 m hohen Dietrich Paß die Brooks-Range quert. Entlang des Dietrich- und Koyukuk Tals verläuft die Trasse nach Süden durch die Hügelketten des Yukon-Tanana Hochlandes. Der Yukon wird etwa $200 \mathrm{~km}$ oberhalb der Einmündung des Tanana im schmalen Rampart-Canyon auf einer Brücke gequert, die auch der Straßenführung dient. Die Rohrleitung berührt die Außenbezirke von Fairbanks und kreuzt weiter südlich die Alaska Kette am $1000 \mathrm{~m}$ hohen Isabell $\mathrm{Pa} \beta$, um dann in das CopperRiver-Basin abzusteigen. Nach Überquerung der Chugach Berge $(750 \mathrm{~m})$ erreicht sie schließlich den Endpunkt im Hafen von Valdez durch den schmalen Keystone-Canyon.

Mit den zur Festlegung der Trasse erforderlichen Bodenuntersuchungen wurde bereits 1970 begonnen, indem Bohrungen im Abstand von etwa einem Kilometer vorgenommen wurden. Die Analyse der Bohrkerne diente außerdem der Ermittlung der Testanforderungen für die Belastung der Rohre und der Erarbeitung der Verlegungsmethoden.

Die Rohrleitung hat einen Durchmesser von $121 \mathrm{~cm}$ (48 inch) und weist je nach erwarteter Belastung eine Wandstärke von 11,5 oder $14,3 \mathrm{~mm}$ auf. Die Rohre sind kunststoffbeschichtet und mit einem Kathodenschutz gegen bakteriologische, chemische und elektrolytische Korrosion versehen. Nur etwa die Hälfte der Leitung $(650 \mathrm{~km})$ wird in konventioneller Weise verlegt. In Permafrostgebieten und erdbebengefährdeten Zonen wurde eine überirdische Führung gewählt. Da das Öl in warmem Zustand zutage tritt und auch während des Transports mit $12 \mathrm{~km}$ pro Stunde durch Reibung eine konstante Temperatur von $60^{\circ} \mathrm{C}$ beibehält, würde eine unterirdische Verlegung in Permafrostböden zum Einsinken und eventuellen Bruch der Leitung führen. Sie ruht in diesen Gebieten auf Trägern, die in den Boden eingefroren werden und dort, wo unstabile, d. h. nahe der Frostgrenze liegende Böden vorherrschen, durch Wärmetauscher unter dem Gefrierpunkt gehalten werden.

Bevor mit der eigentlichen Rohrverlegung begonnen werden konnte, wurden umfangreiche Vorarbeiten notwendig. Wichtigste Voraussetzung war der Bau einer ganzjährig befahrbaren Straße entlang der Trasse, um den Material- und Maschinentransport in das ent-

Dr. Hans-J. Aubert und Ulf E. Müller, 53 Bonn/Bad Godesberg, Augustastraße 83, Deutschland 
Abb. 1: Verlauf der Pipeline.

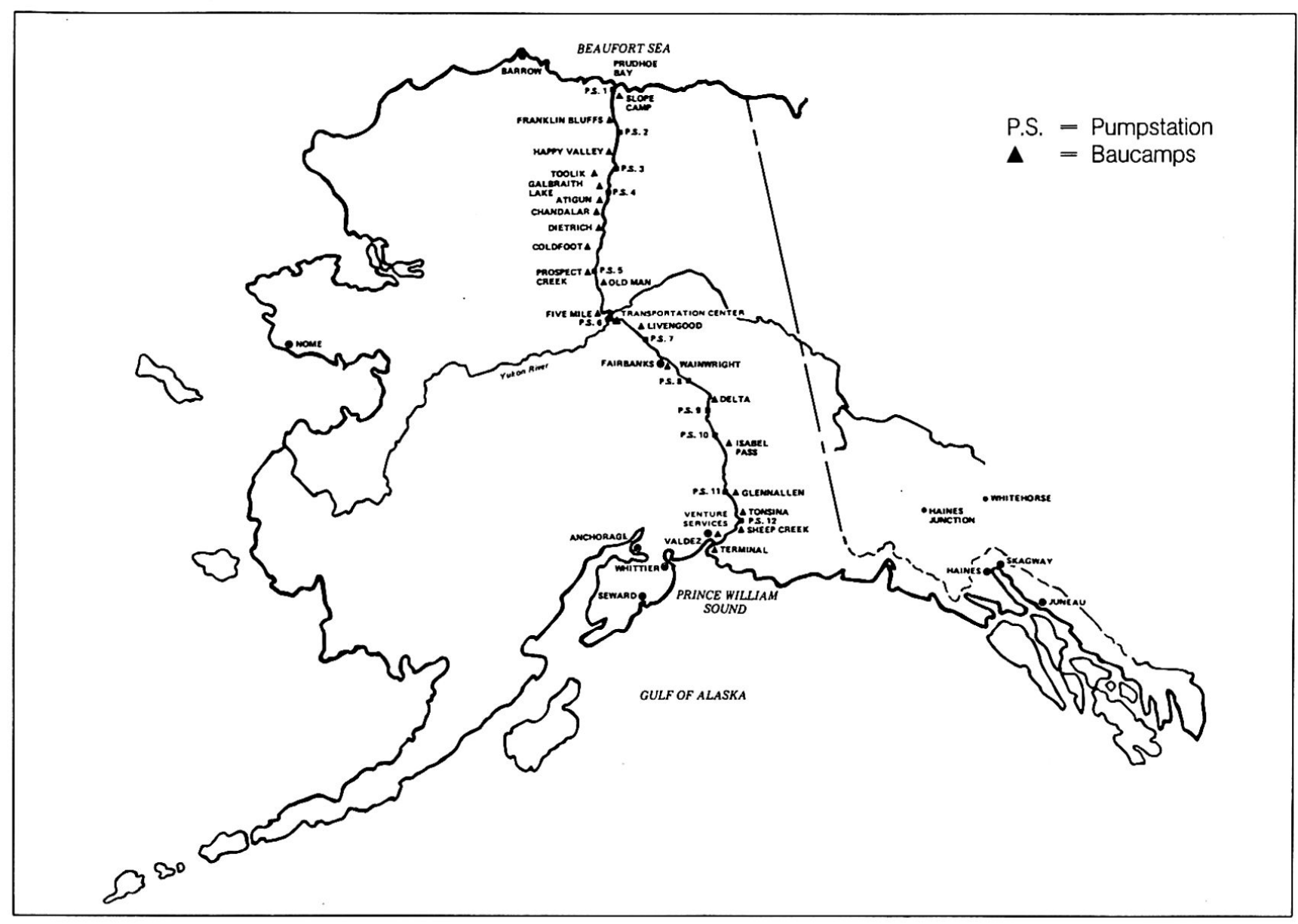

legene Gebiet nördlich des Yukon zu gewährleisten, denn nur südlich von Fairbanks konnte weitgehend auf das bestehende Straßennetz zurückgegriffen werden. Die Arbeit an der $580 \mathrm{~km}$ langen Straße von Livengood nach Prudhoe Bay begann im Dezember 1973 mit der Errichtung einer Eisenbrücke über den Yukon. Bereits im Februar des folgenden Jahres war eine Winterstraße zum arktischen Ozean fertiggestellt, auf der in 83 Tagen $33700 \mathrm{t}$ Ausrüstung und Baumaterial auf acht Baucamps verteilt wurden. Am 29. April begann der Bau der eigentlichen ganzjährig befahrbaren Straße. Während der Sommermonate èfolgte die Versorgung der Baustellen auf dem Luftwege, wobei $160000 \mathrm{t}$ Material und über 30 Mio 1 Treibstoff eingeflogen wurden. Gleichzeitig wurde die Arbeit an den 12 Pumpstationen und den Arbeitscamps vorangetrieben. Am 29. September, 154 Tage nach Baubeginn, war die Straße fertiggestellt, die nach
Beendigung der Verlegungsarbeiten in das Straßennetz des Staates Alaska eingefügt wird. In Valdez und Fairbanks, wo sich die Hauptlager der schon 1969 bestellten Rohre befinden, wurden die Segmente zu $.24 \mathrm{~m}$ langen Einheiten zusammengeschweißt und mit Lastwagen zu den Baustellen befördert.

Sehr sorgfältig wurde darauf geachtet, die Umweltbelastungen auch während der Bautätigkeit so gering wie möglich zu halten. Abfälle wurden teilweise ausgeflogen und Arbeitslager während der Brutzeit seltener Vögel vorübergehend geschlossen.

Mit Beginn der Leitungsverlegung erreichte die Arbeit an der Pipeline ihren Höhepunkt. Bis zu 16000 Menschen waren beschäftigt, über 200 Schwergutlastwagen kreuzten täglich den Yukon zunächst auf Luftkissenfähren, später auf der 1975 fertiggestellten Brücke. Ende 1975 waren 40\% der Rohrleitung verlegt. Während in den Wintermonaten die Arbeit an 
Abb: 2: Staat und Alyeska Pipeline Service Company teilten sich in die Kosten dieser 1975 fertiggestellten Brücke über den Yukon River, welche einmal auch die Pipeline tragen wird. Wir blicken in südlicher Richtung in noch unbesiedelte Waldgebiete. Bisher existierten nur temporäre Brücken im Gebiete des Yukon River.

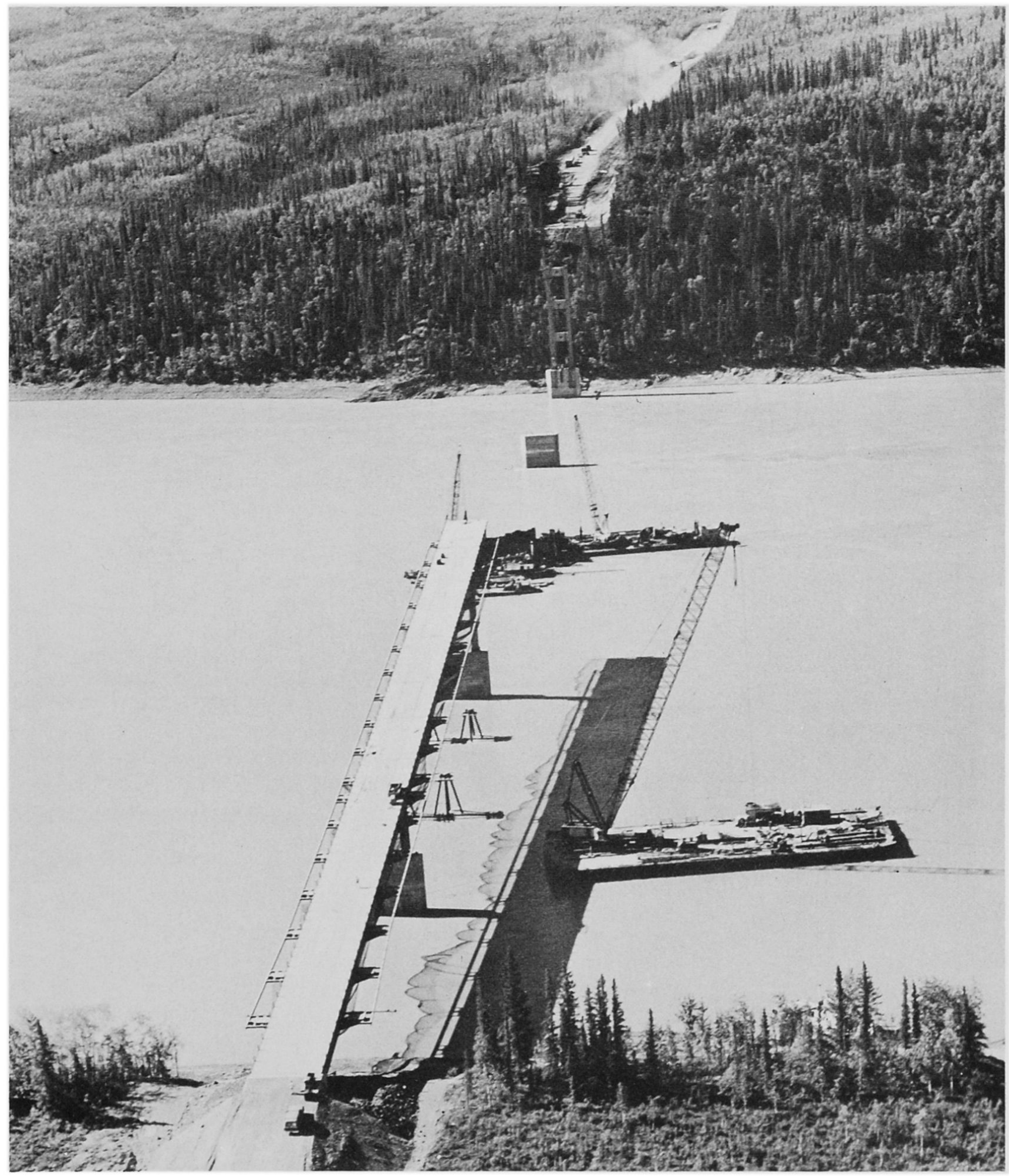


der unterirdischen Verlegung wegen des gefrorenen Bodens ruhen muß, kann der Bau an den überirdischen Teilabschnitten auch in der kalten Jahreszeit fortgesetzt werden.

Besondere Aufmerksamkeit wird naturgemäß der Sicherung der Rohrleitung gegen Erdbeben und der Kontrolle der Dichtigkeit gewidmet. An gefährdeten Stellen ist die Pipeline auf Schlitten gelagert, die horizontale Verschiebungen von bis zu $6 \mathrm{~m}$ und vertikale von bis zu 1,5 m ohne Deformation der Leitung auffangen können. Diese bewegliche Lagerung im überirdisch geführten Abschnitt soll - verbunden mit einer Zickzackführung - auch dazu dienen, die durch Temperaturschwankungen hervorgerufenen Dehnungen und Schrumpfungen auszugleichen. In tektonisch kritischen Gebieten, wie etwa dem Denali-Fault, sind Seismometer angebracht, die in Verbindung mit Ventilen bei Überschreiten eines Schwellenwertes ein automatisches Schließen des gefährdeten Abschnitts bewirken. Der Abstand der Ventile wurde so gewählt, $\mathrm{da} ß$ bei einem Bruch der Leitung der Ölverlust auf 4800001 beschränkt bleibt. Von der Zentrale Valdez können überdies alle Pumpstationen innerhalb von 5 Minuten stillgelegt werden. Der dabei streckenweise auftretende Überdruck wird dann in entlang der Leitung für diesen Zweck errichteten Tanks aufgefangen. Zur laufenden Kontrolle der Dichtigkeit kommen verschiedene Verfahren zur Anwendung. Neben dem Vergleich von Input und Output erfolgen Messungen von Druck, Durchfluß und Volumen, wodurch Schwankungen im Öltransport von weniger als $1 \%$ festgestellt werden können. Sollte es tatsächlich einmal zum Brüch eines Abschnitts kommen, der eine Stilllegung erforderlich macht, bleibt das Öl durch die Isolierung der Rohre bis zu 21 Tagen pumpbar. Im Winter dürte sich dieser Zeitraum jedoch wesentlich verkürzen. Gelingt die Reparatur nicht, bevor das sich noch in der Leitung befindliche Öl erstarrt, muß die gesamte Pipeline aufgegeben werden.

Als schwierig erwies sich auch die Anlage des Endhafens Valdez, der 1964 durch seismische Meereswellen zerstört wurde. Sie hatten ihren Ursprung in einem durch das Erdbeben ausgelösten untermeerischen Erdrutsch loser Sedimente in der Mündung des Lowe River. Um derartigen Gefahren zu begegnen, sind die Lagertanks (32 mit einer Kapazität von 25 Millionen $\mathrm{hl}$ ) über der potentiellen Hochwasserlinie auf gewachsenem Fels errichtet. Die Piers - drei feste und zwei schwimmende - sind so ausgelegt, daß sie Wellen bis zu vier Meter Höhe widerstehen, wenn ein Tanker längsseits liegt. Die Beladung erfolgt ohne Schlauchleitungen und kann in 7 Sekunden unterbrochen werden.

Eine spezielle Regelung mußte auch für den Schiffsverkehr gefunden werden, da der Fjordhafen Valdez nur durch die 5 Meilen breite Hinchinbrook Einfahrt und die $1 / 2$ Meile breiten Valdez Narrows zu erreichen ist. Um Kollisionen zu vermeiden, sind getrennte Einund Ausfahrten vorgesehen, in den Valdez Narrows wechselseitiger Einbahnverkehr. Eine besondere Gefährdung dürfte von diesen Engpässen jedoch nicht ausgehen, da täglich nicht mehr als drei Tanker den Hafen anlaufen oder verlassen. Die speziell für den Transport des Alaska-Öls bereitgestellte Flotte beläuft sich auf 35 Schiffe zwischen 45 und 150 Ts $t$ (dwt), die ihre Ladung in den Raffineriezentren von Puget Sound, San Francisco und Long Beach löschen werden. Das bei der Fahrt nach Valdez mitgeführte Ballastwasser wird in Tanks aufgefangen und nach der Reinigung von Ölrückständen ins Meer zurückgepumpt.

Welche Impulse von der Pipeline auf die wirtschaftliche Entwicklung Alaskas ausgehen, ist derzeit noch kaum abzusehen. Mit dem Bau der Straße zum Eismeer ist eine potentielle Leitlinie wirtschaftlicher Expansion entstanden, von der die Erschließung bisher nicht abbauwürdiger Mineralvorkommen in der Brooks-Range vorangetrieben werden kann. So könnte die vieldiskutierte Alaska-Pipeline weit mehr werden, als ein temporär begrenzter Transportweg für Rohöl vom arktischen Ozean.

\section{Literatur}

Alyeska Reports, quarterly, Jan. 1975 ff, Anchorage. The marine transportation of Alaska North Slope oil, Anchorage, o. D.

Oil spill prevention measures for the Trans-Alaska Pipeline system, Anchorage 1973.

Route map of the Alaska-Pipeline, Anchorage, o.D. Valdez to Prudhoe Bay, Dedication of the State Road Livengood to Prudhoe Bay, Anchorage 1974.

Karte und Bilder: Alyeska pipeline service Comp, Anchorage. 